\title{
Color Changes of Pediatric Dental Bridges
}

\author{
Baydaa Ali Othman Al Rawi ${ }^{1 *}$ and Khalid Hasan Rashash ${ }^{2}$ \\ ${ }^{1}$ Department of Pediatric Dentistry, College of Dentistry, Ibn Sina University of Medical and Pharmaceutical Sciences, Iraq
}

${ }^{2}$ Ministry of Health, Al Anbar health directorate, Iraq

*Corresponding author: Baydaa Ali Othman Al Rawi, Department of Pediatric Dentistry, College of Dentistry, Ibn Sina University of Medical and Pharmaceutical Sciences, Iraq

\begin{abstract}
Dental technology that depended on the standardized lost-wax casting technology has been greatly improved with the introduction of dental CAD / CAM systems. The aim of the present study was to compare between the color changes of CAD/CAM acrylic and manually performed acrylic bridges used for pediatric patients. Forty study casts of children aged 2 - 4 years old of both genders, with prematurely lost one of the maxillary central incisors and the adjacent lateral incisor was carious and considered to be abutment tooth were involved in this study for construction of cantilever bridges. For each cast, two bridges were constructed; $1^{\text {st }}$ one is CAD/CAM acrylic bridge and the other one is manually performed acrylic bridge. After immersing the bridges in saturated chocolate solution for different time intervals, color changes of the bridges were measured using 3Shape scanner system.
\end{abstract}

Keywords: Color; Changes; CAD/CAM; Bridges; Pediatric; Patient

\section{Introduction}

Trauma and/or dental caries is the common causes those result in premature loss of teeth in children. Cosmetic/aesthetic restoration of such condition considers to be challenging in the pediatric dental field. In case of premature tooth loss in anterior incisal segment there will result in arch space loss and teeth's inclination that causing a collapse of the anterior teeth and midline shifting [1], as well as may lead to parafunctional habits [2]. Mahmoud (2009) found that anterior tooth loss had effect on patient's quality of life and gave negative effects on him/his [3]. Al Rawi (2017) found that placement of cantilever acrylic bridges for restoring the aesthetic dental appearance of preschool children resulted in positive successes both to the child and parents [4]. Extrinsic discoloration of teeth and oral prostheses is stains caused by foods or beverages. In pediatric patients such stain mostly occurred due to colored foods such as beets or chocolate as well as berries and candies $[5,6]$. This study considered to be the first step of our series studies deal with determining different physical and mechanical properties of the prostheses used for pediatric patients we planned to carry out (in vitro and in vivo studies). Starting with the present study that aimed to compare between the color changes of CAD/CAM and manually performed acrylic bridges used for pediatric patients. After immersing the bridges in chocolate solution for different time intervals, color changes of the bridges were measured using 3Shape scanner system.

\section{Material and Methods}

This study starting with collection of forty study casts of children aged 2-4 years old of both gender, with prematurely lost one of the maxillary central incisors and the adjacent lateral incisor was carious and considered to be abutment tooth were involved in this study for construction of cantilever bridges (Figure 1). For each cast, two bridges were constructed; 1 st one is CAD/CAM acrylic bridge and the other one is manually performed acrylic bridge (Figure 2). Construction CAD/CAM bridge: The cast was 3D scanned by special scanner (710 3D) (smart optics Sensortechnik GmbH, Germany). The design of the bridge was carried out using Exocad Program (smart optics Sensortechnik GmbH, Germany). Acrylic block (Poly-methyl methacrylate) of classic shade A1 (Ivoclar vivadent, Switzerland) was used for fabrication of the bridge using CAD/ CAM machine (Charly dental, ZI Fonlabour, France). The bridge was finished and polished very well [4]. Construction of manually acrylic bridge: Wax pattern was fabricated on cast then followed the technique of typical wax loss; the heat-cure acrylic (Ivoclar vivadent, AG, FL-9494 Schaan/Liechtenstein) of classic shade 
A1 was used for bridge fabrication. Finally, surface finishing and polishing was done [7]. Saturated chocolate solution was prepared using 15g chocolate powder (MacChocolate TM, Malaysia) with $100 \mathrm{ml}$ distilled water. Baseline color readings for acrylic bridges were taken then immersed in chocolate solution for different time intervals (one week and two weeks) and maintained in incubator of $37{ }^{\circ} \mathrm{C}$, Fresh chocolate solution was prepared every day. Before color measurements after one week and two weeks' time intervals, the bridges were rinsed with distilled water for 30 seconds, cleaned with a soft bristle toothbrush and then dried with tissue paper [8]. Color measurement was carried out in the facial surfaces at the center third of the abutment and the center third of the pontic part of each bridge as shown in Figure 3. Color measurements of the bridges were measured using 3Shape scanner system (3 Shape A/S, Holmens Kanal 7.1060 Copenhagen K Denmark) and according to the software program of the system, Classic shade (Ivoclar vivadent, Switzerland) was depended.

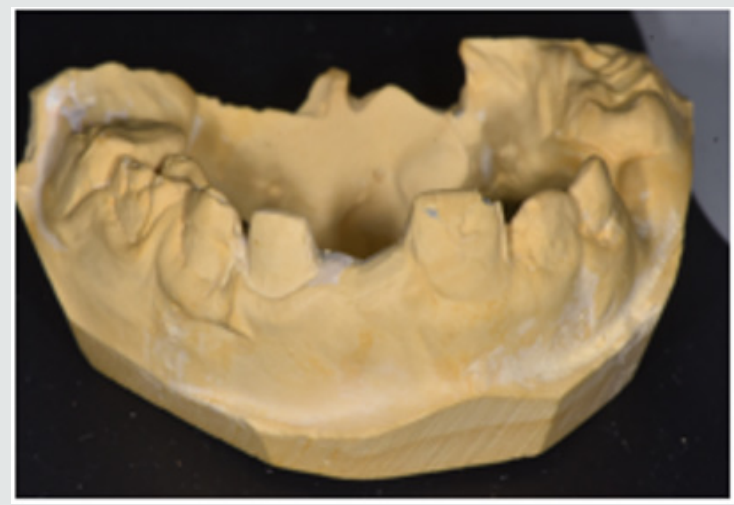

Figure 1: One of the study casts involved in this study.

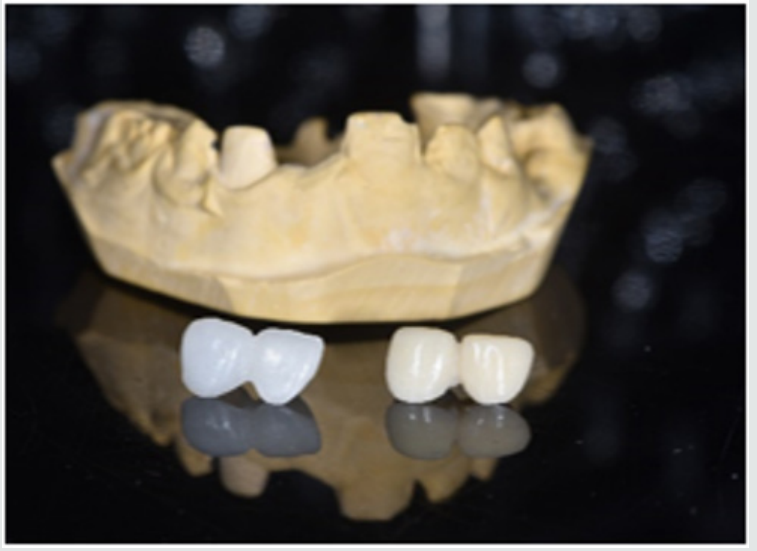

Figure 2: CAD/CAM acrylic bridge and manually performed acrylic bridge for each cast involved in this study.

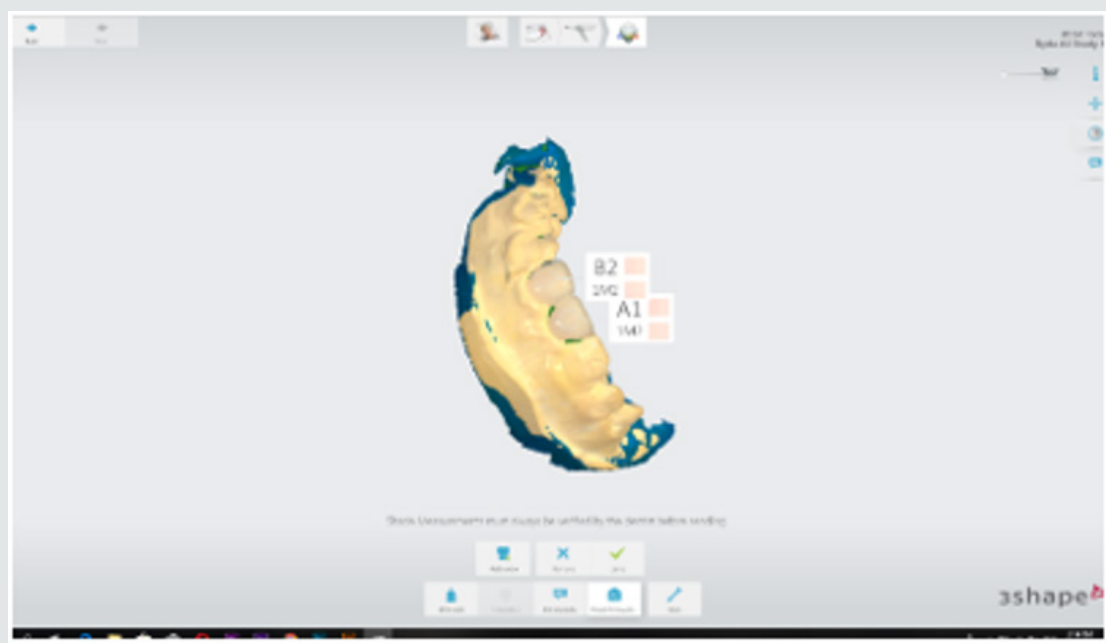

Figure 3: Demonstrated the color shade measurement of the abutment and pontic portions of the acrylic bridge. 


\section{Results}

Table 1 demonstrated the color shade of all samples at the baseline and after one-week and two weeks-time intervals. The results of the present study revealed that for all samples, the color measurement demonstrated that in CAD/CAM group even with using A1 shade acrylic block but at the baseline measurement the abutment revealed $\mathrm{A} 0$ shade while the pontic revealed $\mathrm{A} 1$ shade.
Meanwhile, in manual group the abutment measured to be A1 shade and the pontic gave $\mathrm{B} 1$ shade. The results demonstrated that for all samples there were no changes in the color shade of CAD/CAM and manually fabricated acrylic bridges after one-week time interval, meanwhile, there were significantly color changes of all abutment and pontic portions of all samples of both bridge types after two weeks-time interval immersed in chocolate solution (Figure $4 \& 5$ ).

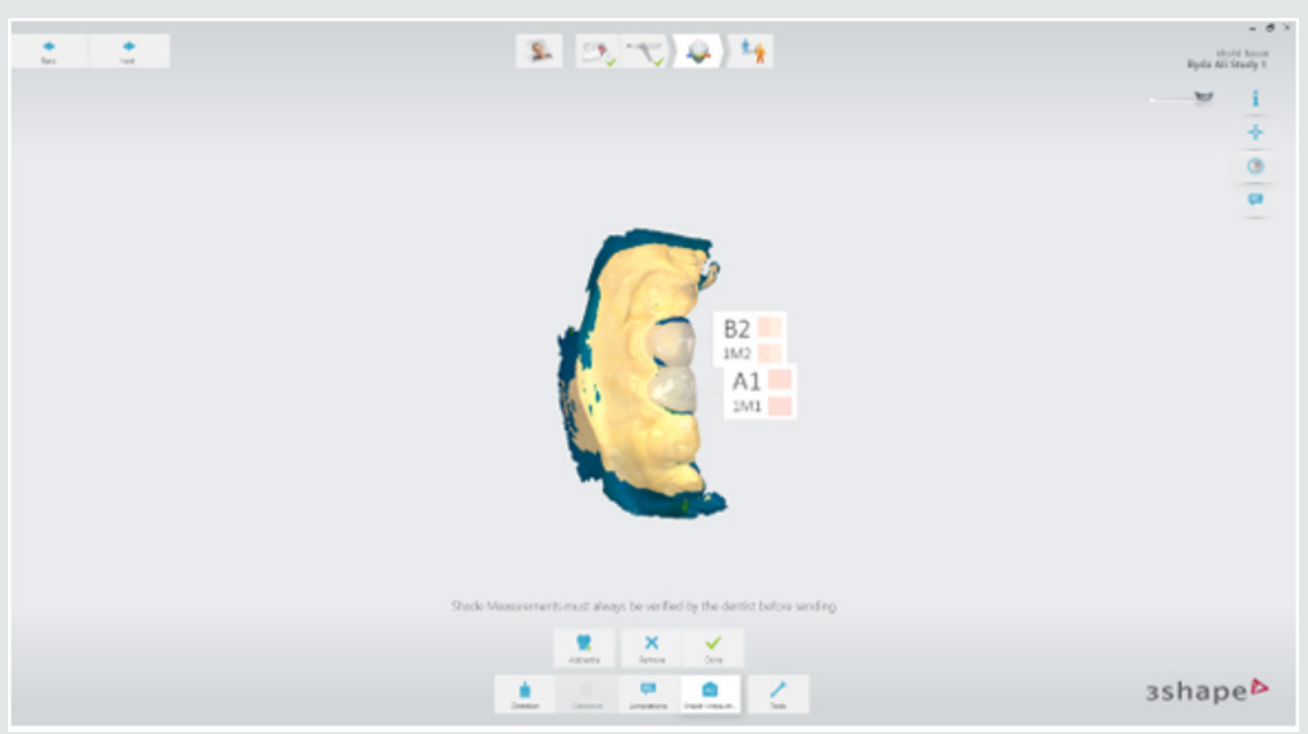

Figure 4: color shade measurement of CAD/CAM acrylic bridge after two weeks.

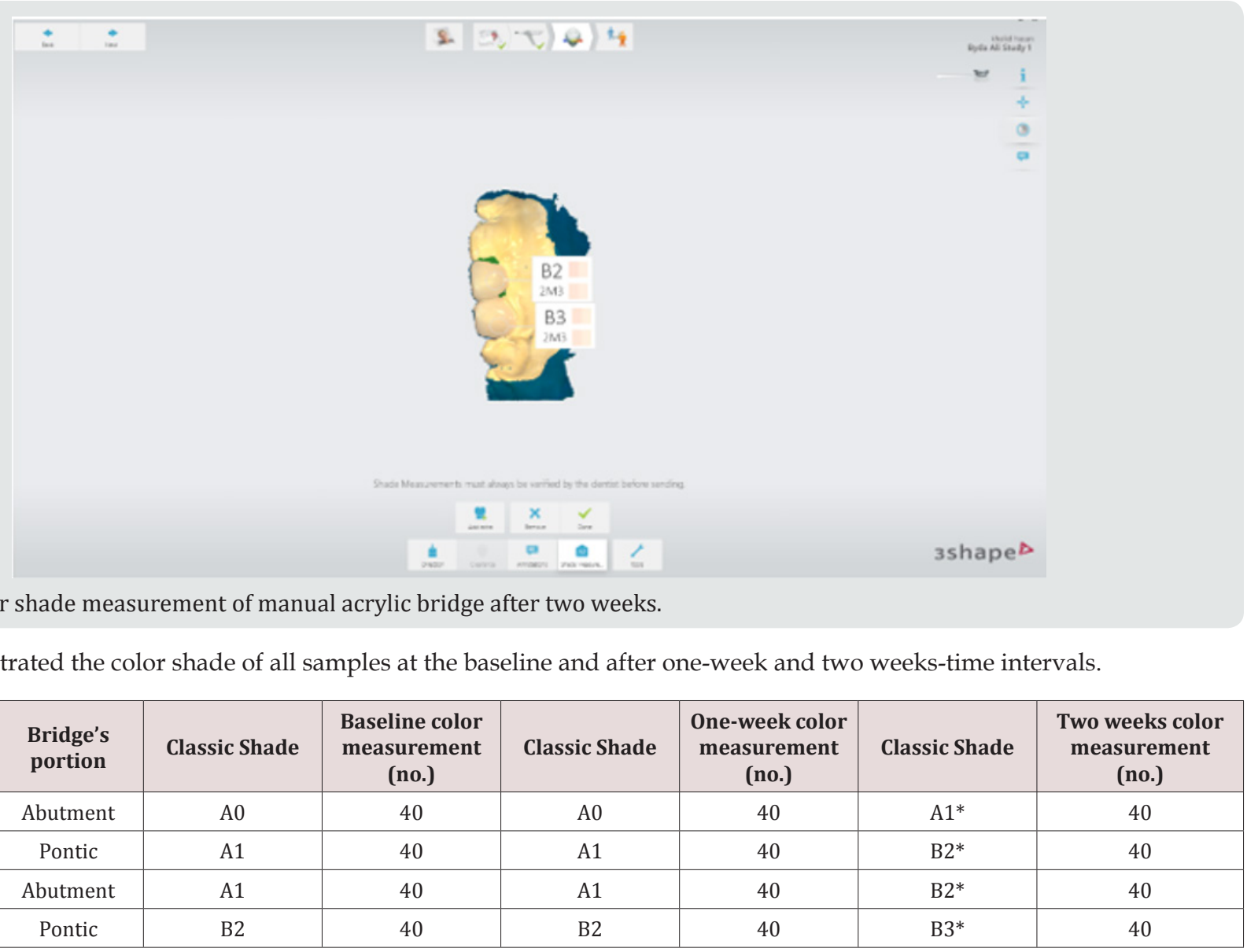




\section{Discussion}

Restorations in the oral cavity are exposed to several factors that make them vulnerable to color changes, such as temperature, humidity, food and beverages. In the oral environment, restorative materials are also subjected to numerous other liquids, to temperature and load stress, and to tooth brushing. The success of restorations depends not only on mechanical and physical properties, but also on the esthetic appearance [9]. The color measurement in this study demonstrated that in CAD/CAM group even with using A1 shade acrylic block but at the baseline measurement the abutment revealed $\mathrm{A} 0$ shade while the pontic revealed A1 shade. These occurred because the thickness of the abutment was only about $0.5 \mathrm{~mm}$ lead to that the color measured of the abutment was lighter than the pontic portion. Meanwhile, in manual group the abutment measured to be A1 shade and the pontic gave B1 shade. These results agreed with other studies those found the thickness of the material significantly affected the color shade of the prostheses $[10,11]$.

The results demonstrated that the color changes demonstrated only after two weeks-time intervals immersed in chocolate solution. Even the color shades recorded in the CAD/CAM group considered to be lighter than in manual fabricated group, the discoloration from chocolate solution was probably due to adsorption of color colorant of chocolate solution at the surface of the prostheses.

The CAD/CAMbridges fabricated from blocks ofpre-polymerized acrylic resin those had a hydrophobic surface that repels water [12]. As well as, perfect polishing surfaces of the bridges involved in this study revealed the limited discoloration that occurred agreed with other research [13]. As the duration of immersion increased, the color change values of both types of prostheses were recorded by 3 Shape scanner system. Thus, the time is considered to be important factor in the staining of the dental prostheses and these results agreed with others [14,15]. Fabrication of dental prostheses with the help of CAD/ CAM technology is related to the advantages of high-density polymers based on highly cross linked polymethylmethacrylate [16]. Those advantages include; good esthetic, low water solubility and absorption, sufficient strength, low toxicity, easy repair with simple fabrication technique [17]. The using of hot cure acrylic for fabrication of dental prostheses even of some advantages but the main disadvantages include porosity with the presence of residual monomer which is a potential allergen, increased finishing time, brittle and uneven thickness [18]. A limitation of this study is that it was an in vitro study and need to be collected with in vivo study to measure the degree of color changes of the prostheses with presenting the effect of saliva and oral hygiene measures. Further clinical and in vitro studies are necessary to evaluate the susceptibility of CAD/CAM and manually acrylic bridges to discoloration by other beverages and nutrients.

\section{Conclusion}

Color considered as the most important factors for aesthetic appearance of dental restorations. In addition to the optimal chemo mechanical properties of acrylic resins, their availability in different color-shades has increased their application in fixed and removable prostheses. Acrylic resins can have acquired discoloration over time because of the process of adsorption and liquid molecules adhere to resin materials which was decreased their effect with using of CAD/CAM technology over conventional methods of acrylic resin prostheses fabrication.

\section{References}

1. Tandon S (2008) Textbook of pedodontics. (2 ${ }^{\text {nd }}$ edn), Paras Medical Publisher pp. 446-465.

2. Da Silva K, Roy B, Yoon RK (2012) Early loss of primary incisors due to para-functional tendency. New York State Dent J 94(11): 38-41.

3. AL Omiri MK, Karasneh JA, Lynch E (2009) Impacts of missing upper anterior teeth on daily living. Int Dental J 59(3): 127-132.

4. Al Rawi BA (2017) Multi-Benefits of CAD/CAM Acrylic Bridge for Primary Incisors. Global Advanced Research Journal of Medicine and Medical Sciences 6(10): 245-248.

5. Vignarajah S (1997) Oral health knowledge and behaviours and barriers to dental attendance of school children and adolescents in the Caribbean island of Antigua. International Dental Journal 47(3): 167-172.

6. Tunc ES, Bayrak S, Guler AU, Tuloglu N (2009) The Effects of Children's Drinks on the Color Stability of Various Restorative Materials. Journal of Clinical Pediatric Dentistry 34(2): 147-150.

7. Jathar P, Panse A, Desai AR (2018) Acrylic crowns for esthetic rehabilitation of primary teeth. International Journal of Pedodontic Rehabilitation 3(1): 42-45.

8. Haselton DR, Diaz Arnold AM, Dawson DV (2005) Color stability of provisional crown and fixed partial denture resins. J Prosthod Dent 93: 70-75.

9. Della Bona A, Kelly JR (2008) The clinical success of all-ceramic restorations. J Am Dent Assoc 139: 8-13.

10. Schaaf NG (1970) Color characterizing silicone rubber facial prostheses. J Prosthet Dent 24(2): 198-202.

11. Ranabhatt R, Singh K, Siddharth R, Tripathi S, Arya D (2017) Color matching in facial prosthetics: A systematic review. J Indian Prosthodont Soc 17: 3-7.

12. Rahal JS, Mesquita MF, Henriques GE, Nobilo MA (2004) Surface roughness of acrylic resins submitted to mechanical and chemical polishing. J Oral Rehabil 31: 1075-1079.

13. Hilgenberg SP, Orellana Jimenez EE, Sepúlveda Navarro WF, Arana Correa BE, Alves TDC, et al. (2008) Evaluation of surface physical properties of acrylic resins for provisional prosthesis. Mat Res 11: 3 .

14. Singh SV, Aggarwal P (2012) Effect of tea, coffee and turmeric solutions on the colour of denture base acrylic resin: an in vitro study. J Indian Prosthodont Soc 12(3): 149-153.

15. Mousavi S, Narimani S, Hekmatfar S, Jafari K (2016) Color Stability of Various Types of Acrylic Teeth Exposed to Coffee, Tea and Cola. J Dent Biomater 3(4): 335-340.

16. Andreescu CF, Ghergic DL, Botoaca O, Barbu HM, Cernusca Mitariu IS, Patroi dn (2017) The Advantages of High-density Polymer CAD/CAM Interim Restorations in Oral Implantology. Mat. Plast 54(1): 32.

17. Urechescu H, Pricop M, Pricop C, Mateas M, Natanael, S, et al. (2017) Thermoplastic Materials Used for Fabrication of Maxillary Obturator Prostheses. Mat Plast 54(3): 477.

18. Bechir A, Pacurar M, Bechir ES, Comaneanu MR, Cires MC, et al. (2014) Aesthetic Importance of Resin based Dental Materials used for Orthodontic Appliances. Mat Plast 51(1): 57. 


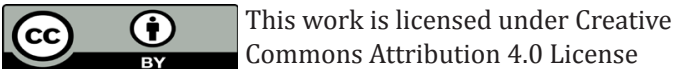

To Submit Your Article Click Here: Submit Article

DOI: $10.32474 /$ IPDOAJ.2019.03.000151

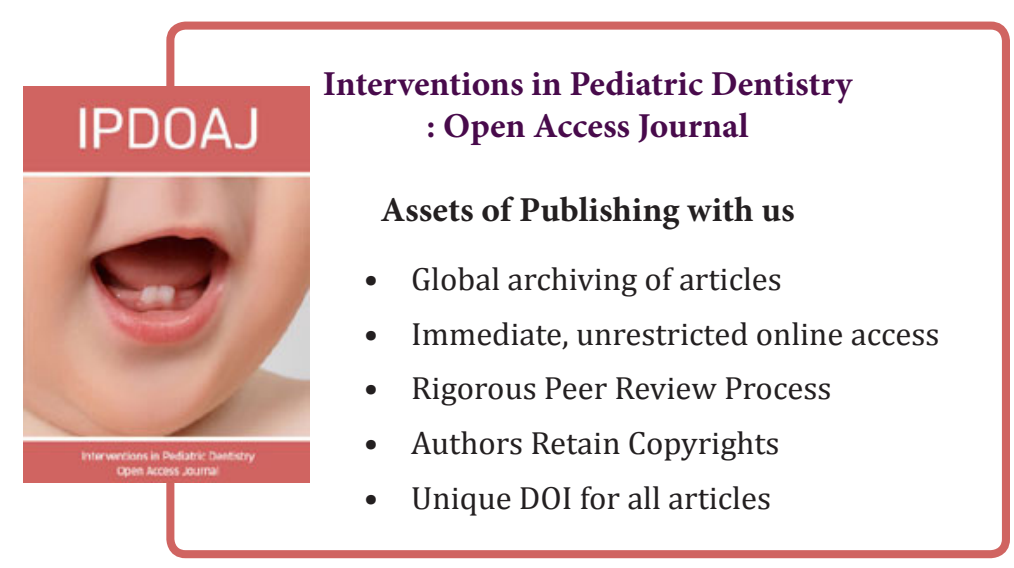

